Belo Horizonte, v. 13, n. 3, p. 63-78, set.-dez. 2020 - ISSN 1983-3652

DOI : $10.35699 / 1983-3652.2020 .24933$

USO DE APLICACIONES ONLINE PARA PLATAFORMAS EN STREAMING:

LANGUAGE LEARNING WITH NETFLIX

ONLINE STREAMING APPLICATIONS AS A PEDAGOGICAL TOOL: LANGUAGE
LEARNING WITH NETFLIX

USO DE APLICATIVOS ONLINE PARA PLATAFORMAS DE TRANSMISSÃO:

LANGUAGE LEARNING WITH NETFLIX

\author{
Silvia Martínez Martínez \\ Universidad de Granada, Espanha \\ smmartinez@ugr.es \\ Coral Ivy Hunt Gómez \\ Universidad de Sevilla, Espanha \\ coralhuntg@us.es
}

RESUMEN: La innovación en el aula y la conexión con los intereses y necesidades del alumnado resultan fundamentales. Así, se examinan los retos metodológicos de la enseñanza CLIL (Content Language Integrated Learning) combinada con la subtitulación accesible de contenido audiovisual de Netflix y la extensión de Google Chrome Language Learning with Netflix. Con objeto de comprobar su eficacia en la mejora de competencias tecnológicas, lingüísticas y el grado de interés mostrado por el aprendizaje de segundas lenguas, se realizó una actividad en la que participaron 75 estudiantes de la Facultad de Traducción e Interpretación de la Universidad de Granada. Los resultados, extraídos a través de un cuestionario realizado exprofeso evidenciaron diferencias entre las diferentes destrezas analizadas, destacando la mejora en la comprensión oral. Además, se pone de relieve que el uso de la metodología en la que se integra la subtitulación para sordos, la metodología CLIL, el aprendizaje de lenguas con fines específicos y el uso de tecnologías de plataformas de contenido audiovisual en streaming mejora el interés y motivación entre el alumnado de educación superior, siendo una herramienta óptima para los docentes que quieran innovar y transcender el aula.

PALABRAS CLAVE: Nuevas tecnologías. Subtitulación para sordos. Enseñanzaaprendizaje. CLIL. Entornos multimodales.

ABSTRACT: Classroom innovation and fulfilling students' needs are paramount for education. Therefore, the methodological challenges of CLIL (Content Language Integrated Learning) teaching combined with the accessible subtitling of Netflix audiovisual content and the extension of Google Chrome Language Learning with Netflix are examined. In order to verify its effectiveness in improving technological and linguistic competences and the degree of interest shown in learning second languages, a pilot study was carried out in which 75 students from the Faculty of Translation and Interpretation of the University of Granada participated. Results from a questionnaire carried out specifically, showed differences between the various skills analysed. The improvement in oral comprehension is the most remarkable. The use of the methodology that integrates subtitling for the deaf and hard-of-hearing, the CLIL methodology, the learning of 
languages for specific purposes and the use of technologies of audiovisual content platforms improves in streaming increase interest and motivation among higher education students. It can be concluded that it is an optimal tool for teachers who want to innovate and go beyond the classroom.

KEYWORDS: New technologies. Subtitling for the deaf and hard-of-hearing. Teaching and learning process. CLIL. Multimodal environments.

RESUMO: A inovação em sala de aula e a conexão com os interesses e necessidades dos alunos são essenciais. Assim, os desafios metodológicos do ensino CLIL (Content Language Integrated Learning) combinados com a legendagem acessível de conteúdo audiovisual da Netflix e a extensão do Google Chrome Language Learning with Netflix são examinados. Para verificar a sua eficácia na melhoria das competências tecnológicas e linguísticas e o grau de interesse demonstrado pela aprendizagem de outras línguas, foi realizada uma atividade com 75 alunos da Faculdade de Tradução e Interpretação da Universidade de Granada. Os resultados, extraídos por meio de questionário profissionalizante, mostraram diferenças entre as diferentes habilidades analisadas, destacando a melhora na compreensão oral. Além disso, destaca-se que a utilização da metodologia que integra a legendagem para surdos, a metodologia CLIL, a aprendizagem de línguas para fins específicos e a utilização de tecnologias de plataforma de streaming de conteúdo audiovisual melhora o interesse e a motivação entre os alunos do ensino superior, sendo uma ótima ferramenta para professores que desejam inovar e transcender a sala de aula.

PALAVRAS-CHAVE: Novas tecnologias. Legendagem para surdos. Ensinoaprendizagem. CLIL. Ambientes multimodais.

\section{Introducción}

En los últimos años, la sociedad globalizada ha experimentado un aumento exponencial del consumo de contenidos audiovisuales multilingües gracias a la aparición de diferentes industrias que los ofrecen en streaming en plataformas transnacionales. Una de ellas, Netflix, alcanzó los 167 millones de usuarios a finales de 2019 (AGENCIA AFP, 2020) y goza de una creciente popularidad, en especial, entre los jóvenes. Las plataformas de vídeo bajo demanda por suscripción están integradas en la cotidianidad de una gran parte de la población y, como consecuencia, las herramientas digitales y tecnológicas de las que se acompañan están comenzando a integrarse progresivamente en distintos contextos educativos, a pesar de que, en muchos casos, su uso y aplicación pueda mejorarse (ALBIO, 2011, p. 34).

Un ejemplo del traspaso de herramientas digitales arraigadas en el ámbito lúdico al de la educación es el uso de subtítulos en el aprendizaje de lenguas por parte de normoyentes. En su origen, la subtitulación estaba destinada a personas con discapacidad auditiva, pero, en la actualidad, todo tipo de usuarios se beneficia de ella para acceder a contenidos en distintos idiomas e incluso se utiliza como herramienta de ayuda al aprendizaje de segundas lenguas o mejora de alguna competencia lingüística concreta. Tanto es así, que, desde 1985, existen en España métodos de aprendizaje de segundas lenguas que incluyen la subtitulación como herramienta didáctica como, por 
ejemplo, Speak Up de la editorial RBA para la lengua inglesa, o Das Rad de la editorial Scholastic Inc. para la lengua alemana. En esta misma línea, diversos autores abogan por el uso tanto de la subtitulación como de otras modalidades de traducción audiovisual en el ámbito educativo (DÍAZ-CINTAS, 2012; TALAVÁN ZANÓN et al., 2017; TALAVÁN ZANÓN, 2019).

Dentro de estas aplicaciones y en un enfoque muy cercano a las últimas tendencias educativas en aprendizaje de segundas lenguas, Fernández-Costales (2017) sugiere la combinación de la metodología CLIL (Content Language Integrated Learning) con el uso pedagógico de los subtítulos y enumera sus múltiples ventajas a nivel de competencias que abarcan desde las puramente lingüísticas hasta las relacionadas con aspectos de bilateralidad o culturales.

Teniendo en cuenta todo lo anterior, este trabajo pretende vincular el uso de nuevas tecnologías dentro de las plataformas de contenido audiovisual con la realidad del aula de idiomas mediante el uso de la subtitulación intralingüística (sonidos y música) e interligüística (diálogos) como herramienta didáctica. Para ello, se analiza la utilidad de la metodología CLIL combinada con la subtitulación accesible de contenido audiovisual de Netflix y la extensión de Google Chrome Language Learning con Netflix. Esta última es una aplicación gratuita fácil de usar y que implica el uso de materiales reales, por lo que se ajusta a los preceptos de utilidad, realidad y propósito de la metodología Content and Language Integrated Learning (COYLE, 2008). Además del uso de materiales auténticos, otra ventaja de la presente metodología docente consiste en permitir a los estudiantes trabajar simultáneamente con su lengua materna (L1) y una lengua extranjera (L2) de manera que despierte el interés y la motivación del alumnado. Uno de los propósitos de esta investigación es analizar la relación entre la aplicación didáctica del software descrito anteriormente y la evolución experimentada por los estudiantes en términos de gramática y vocabulario, así como la mejora de su comprensión lectora y auditiva. Igualmente, se estudia su influencia en la motivación y el interés de los estudiantes. Con este propósito, se cuenta con una muestra de 75 estudiantes de la Universidad de Granada (España) que aprenden inglés como L2, con un nivel intermedio, equivalente a un B1-B2 del Marco Común Europeo de Referencia para las Lenguas (MCER) (CONSEJO EUROPEO, 2001). La tarea que los participantes debían realizar consistió en ver dos episodios de la serie estadounidense Santa Clarita Diet (FRESCO, 2017) en versión original y con subtítulos en inglés para sordos. Posteriormente, estos tenían que cumplimentar un cuestionario que contaba con una parte introductoria destinada a recopilar datos generales y personales de los participantes y tres bloques principales que pretendían obtener información concerniente a las competencias tecnológicas, lingüísticas y al grado de motivación experimentado por los estudiantes.

En un primer apartado se estudia el uso de la subtitulación como herramienta para enseñar segundas lenguas destancando los beneficios que reporta para el alumnado. Tras esa breve contextualización, se detalla el método y los objetivos que se persiguen, al igual que el proceso de elaboración y validación de los instrumentos de medida y del análisis de los datos recogidos. Los resultados se analizan teniendo en cuenta tres dimensiones: la percepción de la mejora de las competencias tecnológicas, la de las competencias lingüísticas y el interés. En último lugar, se exponen las principales conclusiones del estudio, sus limitaciones y se plantean futuras líneas de investigación.. 


\section{La subtitulación en la didáctica de lenguas extranjeras}

La enseñanza de segundas lenguas es una disciplina que ha sufrido una gran evolución tecnológica a lo largo de las últimas décadas. Tal como señalan MartínezMartínez y Álvarez de Morales Mercado (2017, p. 427), "hoy en día ya no se tiende a utilizar los métodos tradicionales de enseñanza, sino que gracias a la evolución digital, se hace uso cada vez más de las nuevas tecnologías". De este modo, la enseñanza de lenguas actualmente está utilizando diversos entornos multimodales como herramienta didáctica, tales como la subtitulación para normoyentes (TALAVÁN ZANÓN; ÁVILA CABRERA, 2015) o las modalidades de traducción accesible: audiodescripción para personas con disfunción visual (MARTíNEZ-MARTÍNEZ, 2012) y la subtitulación para sordos (SpS).

Así pues, el desarrollo de la enseñanza de idiomas desde la accesibilidad es un tema de investigación en auge, en este caso, utilizando el SpS como instrumento metodológico. Los primeros estudios sobre las ventajas del uso de la subtitulación para aprender idiomas se centraron en su influencia sobre diversas competencias. Destacan sus efectos positivos sobre la motivación del alumnado (VANDERPLANK, 1998), los beneficios para el aprendizaje de vocabulario (BIRD; WILLIAMS, 2002), la mejora de la comprensión lectora (BRAVO CODINHO, 2008) y de la capacidad de escucha y memorización (CAIMI, 2006).

Más recientemente y de la mano de la revolución tecnológica y la democratización del acceso a los contenidos audiovisuales en línea, varios autores orientan el uso de la SpS como herramienta para mejorar las destrezas orales y escritas dentro de la enseñanza reglada (TALAVÁN ZANÓN et al., 2017) o, en un ámbito de educación superior, como procedimiento para aprender alguna modalidad de traducción especializada (MARTÍNEZ-MARTÍNEZ; ÁLVAREZ DE MORALES MERCADO, 2017). Dentro de la literatura sobre el uso de subtitulación como herramienta con fines didácticos, Talaván Zanón; Ávila Cabrera (2015, p. 222) indican que, a la hora de subtitular, se pueden presentar carencias lingüísticas tanto en entornos académicos como en contextos profesionales. Así pues, se propone que el alumnado sea capaz de adquirir las destrezas orales, lectoras, auditivas y escritas para que este pueda desenvolverse sin problemas en la vida diaria. Asimismo, esta novedosa metodología podrá servir para reforzar conocimientos referentes al mundo de la traducción e interpretación en su vertiente didáctica. En palabras de Díaz-Cintas (2012, p. 95):

[...] se abre con una panorámica del papel que la traducción audiovisual juega en la didáctica de idiomas: introduce variedad, da cabida a los elementos no-verbales de la comunicación y, lo más importante, permite a los estudiantes ver el tipo de interacción que se establece entre lengua y cultura en un contexto real.

Entre las ventajas del uso de la subtitulación en el aula de enseñanza de lenguas y, de manera especial, cuando se siguen los parámetros generales de la metodología CLIL cognición, cultura, contenido y comunicación (COYLE, 2008) -, destaca la familiarización y la cotidianidad del uso de subtítulos entre los alumnos, ya que influyen en gran medida en la motivación y en el concepto de propósito. Los aprendices son usuarios habituales de subtítulos y para ellos estos recursos accesibles que ofrecen plataformas globales como Netflix son elementos cotidianos que ocupan un lugar dentro sus aficiones. Tampoco hay 
que olvidar que, al tratarse de contenido original y auténtico, el uso de subtítulos encaja a la perfección con la metodología CLIL ya que incluye, entre otros aspectos, el trabajo con elementos culturales y comunicativos.

Diversos estudios recogen los distintos beneficios del uso de la subtitulación como recurso didáctico en el aula CLIL, tales como el desarrollo de la conciencia lingüística de los usuarios y el desarrollo de la capacidad de transferencia de conocimiento inter 0 intralingüística (FERNÁNDEZ-COSTALES, 2017; HUNT-GÓMEZ, 2020). Otro aspecto fundamental es la capacidad integradora de los subtítulos en el aula (DURÁN-MARTÍNEZ; BELTRÁN-LLAVADOR; MARTÍNEZ-ABAD, 2016), puesto que su uso resulta de gran ayuda e incluso imprescindible para aquellas personas que presentan algún tipo de discapacidad auditiva. Según la Organización Mundial de la Salud (2019), en 2050, una de cada 10 personas sufrirá una pérdida de audición discapacitante, y muchas de ellas serán jóvenes, dado que se considera que están en riesgo de pérdida de audición por su sobreexposición al ruido en contextos recreativos.

Por los motivos anteriormente expuestos, se ha utilizado la SpS para realizar una actividad con el fin de comprobar si, efectivamente, esta modalidad de traducción accesible sirve para el aprendizaje de idiomas y, a su vez, resulta adecuada para mejorar los conocimientos lingüísticos, tecnológicos y comunicativos de los estudiantes del Grado en Traducción e Interpretación. Esto integra la metodología CLIL en el aula de educación superior, y hace que vaya más allá del aula de idiomas, recuperando parte de su esencia metodológica que siempre persigue un objetivo concreto.

\section{Método}

Para el desarrollo del estudio se ha seguido una metodología de investigación de carácter cuantitativo a través de un cuestionario. Para ello, esta investigación se ha basado en una actividad piloto en la que se integra un tipo de traducción multimodal, el SpS, para el aprendizaje de lenguas con fines específicos, la metodología CLIL y el uso de tecnologías de plataformas de contenido audiovisual en streaming.

\subsection{Objetivos}

Aunque, de manera transversal esta investigación busca trascender el aula de idiomas, consiguiendo que los estudiantes sigan utilizando estas aplicaciones en contextos no reglados y, a la vez, sensibilizarlos con la comunidad sorda y hacer el aula más inclusiva, los objetivos específicos que se plantean son los siguientes:

1. Comprobar que el uso de la aplicación Language Learning with Netflix y de la SpS para el aprendizaje de segundas lenguas mejora el dominio de las competencias tecnológicas y lingüísticas entre el alumnado de educación superior, y

2. comprobar que el uso de la aplicación Language Learning with Netflix y de la SpS para el aprendizaje de segundas lenguas mejora del interés y la motivación entre el alumnado de educación superior. 


\subsection{Muestra}

Los sujetos que participan en esta investigación son 75 estudiantes del Grado en Traducción e Interpretación que tienen como L2 la lengua inglesa. En este Grado de la Universidad de Granada, la lengua materna (L1) es el español en la mayoría de los casos. Conviene señalar que de los 75 estudiantes encuestados 11 de ellos son estudiantes extranjeros que cursan la ayuda para la modalidad Erasmus. Todos los integrantes de la muestra se encontraban en el momento de la investigación cursando el tercer curso de sus estudios de Grado y contaban con conocimientos previos de gramática de la lengua inglesa y su traducción directa, puesto que habían superado las asignaturas de los cursos anteriores. En el caso de la lengua materna de los sujetos, un 85,33\% $(\mathrm{N}=64)$ de la muestra estaba conformada por hablantes de español, un 1,33\% $(\mathrm{N}=1)$ de hablantes de inglés y un $13,33 \%(\mathrm{~N}=10)$ de otras lenguas. Casi la totalidad de la muestra $(\mathrm{N}=74)$ tenía como primera lengua extranjera (L2) el inglés.

\subsection{Instrumento}

Se ha recurrido al método de la aplicación de un cuestionario que contaba con cuatro dimensiones bien diferenciadas. El cuestionario fue creado exprofeso para recopilar datos de interés y se consideró un procedimiento óptimo puesto que permite recopilar datos reales y fidedignos de forma rápida y eficaz. Para comprobar su validez, el cuestionario fue sometido a juicio de tres expertos pertenecientes a universidades andaluzas. El objetivo específico del cuestionario consistía en recabar información sobre la valoración del uso de las TIC aplicadas a la enseñanza de idiomas y sobre su integración para la mejora de las competencias lingüísticas desde las perspectiva de los estudiantes de grado. Asimismo, cabe señalar que los ítems se redactaron de forma clara, precisa y comprensible, cada uno de ellos se refería a un solo aspecto y que se utilizó un lenguaje simple y directo. La validación del cuestionario se centró en la adecuación de los ítems propuestos a los objetivos perseguidos, en la claridad e inteligibilidad de la redacción y en su pertenencia. Es importante destacar que, para no influir en los resultados del cuestionario, la participación de los sujetos fue voluntaria y anónima.

El instrumento de medida contaba con una parte introductoria destinada a obtener datos demográficos y generales sobre los participantes. Se les plantearon cuestiones sobre la lengua materna y su dominio, sobre su uso de nuevas tecnologías y sobre conocimientos específicos de aplicaciones en streaming para aprendizaje de lenguas o relacionadas con la subtitulación y su uso. Así, se incluian tanto preguntas cortas cerradas y como abiertas, teniendo en cuenta las ventajas e inconvenientes que se podían plantear en cada caso y se seleccionaban las preguntas de acuerdo con el modo de respuesta esperado. El tiempo estimado para su cumplimentación se sitúa alrededor de 15 minutos.

Las dimensiones principales del cuestionario son tres y se enlazan con los objetivos prioritarios del trabajo. La primera dimensión estaba destinada a medir las competencias en nuevas tecnologías de los participantes: si habían tenido algún problema con la instalación de las aplicaciones que se presentaron en el aula, qué aplicación les había resultado más sencilla a la hora de utilizarla y cuál les había gustado más. Por último, tenían que puntuar dichas aplicaciones haciendo referencia a su utilidad. La segunda dimensión medía el aprendizaje de los alumnos en distintos aspectos 
lingüísticos: la gramática, el vocabulario, la comprensión oral, la expresión oral y la comprensión lectora. En este apartado se solicitó a los participantes que ilustraran sus respuestas con al menos un ejemplo. La tercera y última dimensión incluye preguntas sobre la motivación y el interés de los estudiantes tras haber realizado la prueba.

\subsection{Procedimiento}

En una primera fase, dentro de un contexto académico, se familiarizó a los participantes con los rudimentos tecnológicos de las aplicaciones para el correcto desarrollo de la actividad. Una vez completada esta fase, los sujetos tenían que ver el primer episodio de la serie Santa Clarita Diet (FRESCO, 2017) utilizando tres aplicaciones para el aprendizaje de segundas lenguas a través de la subtitulación: Subtly ${ }^{1}$, Sufli ${ }^{2}$ y Language Learning with Netflix ${ }^{3}$. Después, debían ver el segundo episodio de la misma serie utilizando la aplicación que les hubiera parecido más idónea para el aprendizaje de lenguas. El visionado de los episodios se realizó en inglés [V. O.] y con subtítulos accesibles en inglés [CC], según las siglas utilizadas por la propia plataforma de video bajo demanda Netflix. En la siguiente imagen (Figura 1), se puede observar la interfaz de la extensión LLN, así como las opciones de las que dispone.

1 Disponible en: $\quad$ https://chrome.google.com/webstore/detail/subtly-\%E2\%80\%93-subtitles-for-ne/ nldojjdlhkfalipikhhnhhidfhgoopig. Consultado en: 13 nov. 2020.

2 Disponible en: https://chrome.google.com/webstore/detail/sufli-netflix-subtitles-t/ glhnfeapdblacaodpihdfcaijfddedec. Consultado en: 13 nov. 2020.

3 Disponible en: https://chrome.google.com/webstore/detail/language-learning-with-ne/ hoombieeljmmljlkjmnheibnpciblicm. Consultado en: 13 nov. 2020. 
Figura 1: llustración de elección de lenguas de trabajo con LLN.

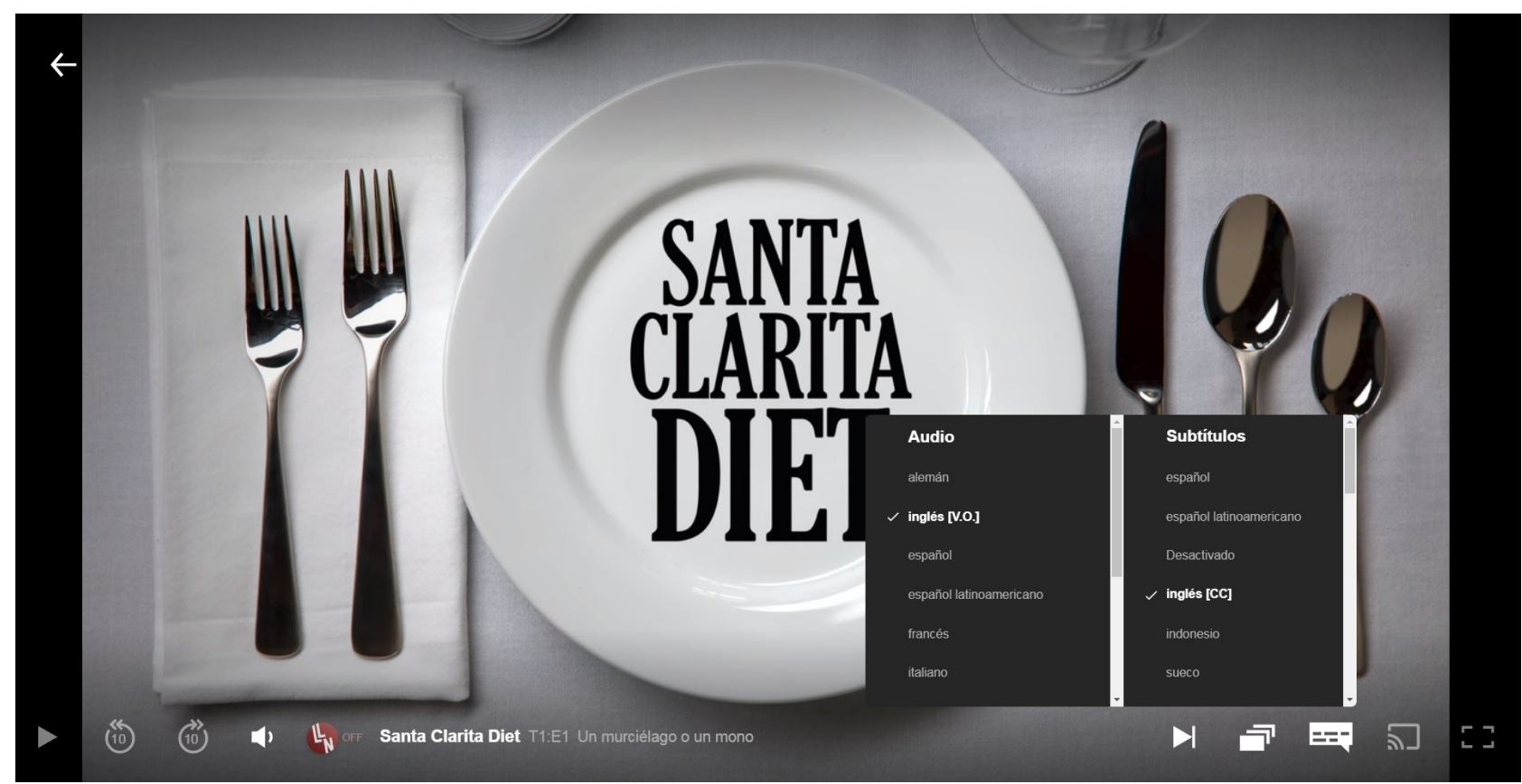

Fuente: Netflix.

Una vez realizada esta tarea, los participantes debían contestar un cuestionario creado exprofeso con el fin de recopilar datos sobre varios aspectos entre los que destacan, la influencia de su uso sobre sus competencias tecnológicas y lingüísticas y su efecto sobre la motivación y el interés.

La participación en la actividad fue estrictamente voluntaria y conforme a la normativa en vigor para la protección de datos personales. El proceso se desarrolló durante el mes de enero de 2020.

\section{Resultados}

A continuación, se presentan los principales resultados obtenidos en la realización de este estudio. La información se ha divido en varios apartados, según la estructuración del cuestionario.

\subsection{Competencias tecnológicas}

Para alcanzar los objetivos establecidos, resulta necesario analizar el tipo de tecnologías con las que los participantes estaban familiarizados previamente. Se puede observar que un $100 \%$ de los sujetos usan teléfonos móviles $(\mathrm{N}=75)$ y que un $80 \%$ usa tabletas $(\mathrm{N}=60)$, además, la gran mayoría de los sujetos afirma ser usuario frecuente de contenido audiovisual entre el que se incluyen vídeos (94\%, $\mathrm{N}=70)$, aplicaciones (100\%, $\mathrm{N}=75)$ y series y/o películas en versión original (75 \%, N=56) (Gráfico 1). 
Gráfico 1: Uso de nuevas tecnologías.

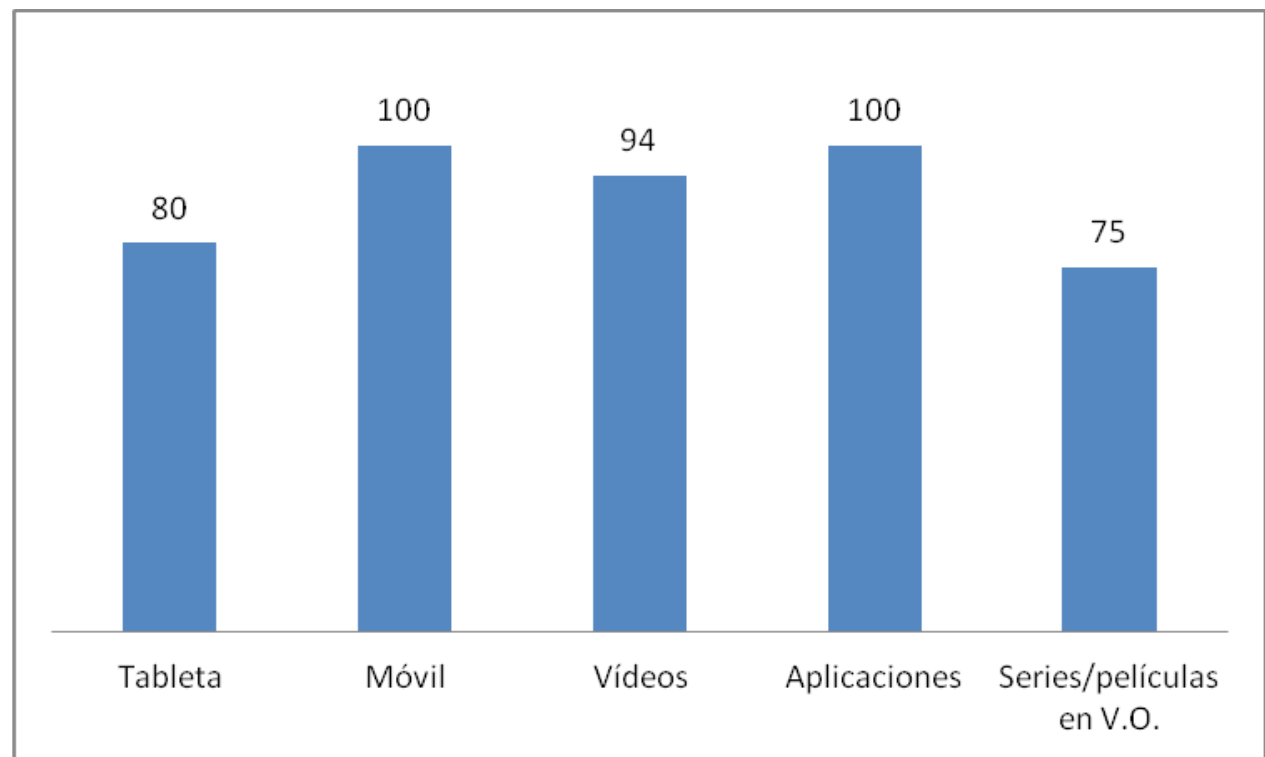

Fuente: elaboración propia.

En cuanto al uso de plataformas de contenido audiovisual en streaming los resultados indican que un $72 \%(\mathrm{~N}=54)$ de los sujetos usaban Netflix, un $60 \%(\mathrm{~N}=45)$ $\mathrm{HBO}$, un $45 \%(\mathrm{~N}=33)$ Amazon Prime y un $10 \%(\mathrm{~N}=7)$ otras plataformas (Gráfico 2). Cabe destacar también, que muchos de ellos usaban dos o tres plataformas de vídeo bajo demanda de manera simultánea.

Gráfico 2: Uso de plataformas en streaming.

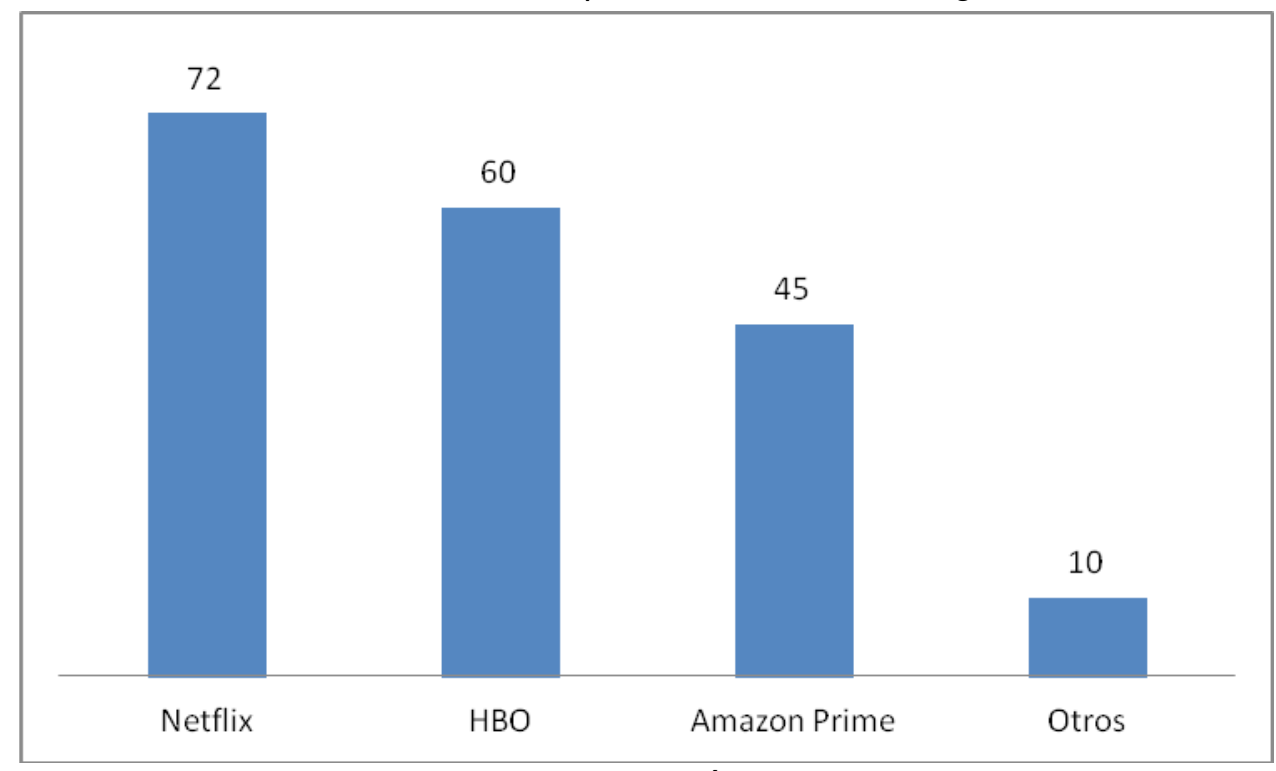

Fuente: elaboración propia.

El análisis de las respuestas sobre el conocimiento previo de las aplicaciones específicas para el aprendizaje de segundas lenguas utilizando los subtítulos como herramienta didáctica: Subtly, Sufli y Language Learning with Netflix revela que 
prácticamente la totalidad de los sujetos desconocía estas aplicaciones, y solo una persona estaba familiarizada con la aplicación Language Learning with Netflix, aunque afirmaba no haberla usado.

De los resultados obtenidos sobre el uso de las plataformas y los dispositivos desde los que se accede a ellas se puede extraer que los sujetos incluyen el uso de teléfono móviles y tabletas como principales dispositivos para ver contenido audiovisual en las distintas plataformas que lo ofrecen. De estas plataformas, casi tres cuartas partes de los sujetos utilizaban Netflix y, muchos de ellos, combinaban su uso con el de otras plataformas. Cabe destacar que, a pesar de estar muy familiarizados con las plataformas de contenido audiovisual y de su uso sea cotidiano, la mayoría de los participantes no conocen sus aplicaciones didácticas para el aprendizaje de lenguas.

Otro aspecto objeto de estudio es la facilidad de uso de las aplicaciones en relación a las competencias tecnológicas de los participantes. En ese sentido, los sujetos señalaron algunos problemas al instalar alguna de las tres aplicaciones. Estas dificultades influyeron en la elección de la aplicación que les pareció más conveniente para ver el segundo episodio, puesto que los resultados indican que tanto Subtly como Sufli generaron problemas informáticos, mientras que Language Learning with Netflix les había resultado más fácil de usar gracias a su carácter intuitivo. Así, los resultados recogen que el $98 \%(\mathrm{~N}=73)$ eligió Language Learning with Netflix, seguida de Subtly con un $1 \%(\mathrm{~N}=1)$ y Sufli con el mismo porcentaje $(1 \%, \mathrm{~N}=1)$. A pesar de que todas ellas son compatibles con la plataforma de contenidos en streaming Netflix, este resultado se puede deber en parte a que Subtly no funciona con todos los sistemas operativos y a que Sufli no dispone de una interfaz fácil de usar, según los comentarios de los encuestados.

Así pues, la mayoría de los participantes decidió utilizar Language Learning with Netflix para el visionado del segundo episodio (We Can't Kill People!). Los principales motivos aducidos por los sujetos para la selección de esta aplicación eran que es más intuitiva que las otras dos y que dispone de una interfaz más familiar, ya que incluso se puede utilizar el teclado para parar un subtítulo o retroceder. Además, tiene la ventaja de poder ver los subtítulos tanto en inglés como en español de forma simultánea (u otro par de lenguas de nuestra elección) y este hecho hace que sea posible ver la traducción, las estructuras gramaticales de ambas lenguas y observar de primera mano la conceptualización de la realidad y léxico relativo a usos y costumbres, aspecto que le ha interesado bastante al perfil encuestado. A modo de ejemplo, se puede ver la siguiente imagen (Figura 2) para comprobar cómo se pueden ver los subtítulos en dos lenguas diferentes. 
Figura 2: Captura de pantalla de la serie Santa Clarita Diet (Fresco, 2017) con la aplicación Language Learning with Netflix.

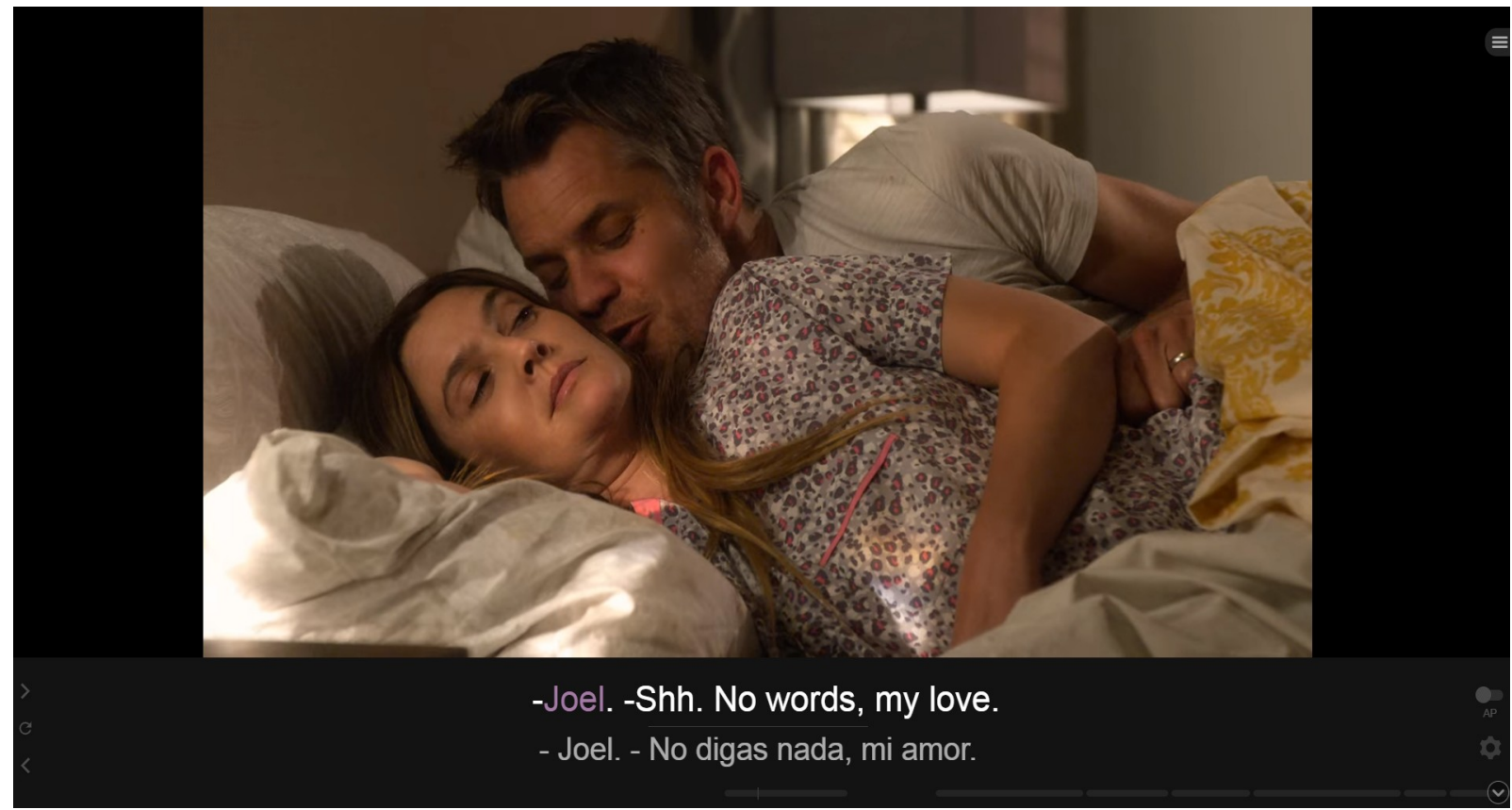

Fuente: Netflix.

\subsection{Competencias lingüísticas}

Otro aspecto fundamental de este trabajo consiste en comprobar si la realización de esta actividad había hecho que las competencias lingüísticas de los sujetos mejoraran.

Gráfica 3: Resultados de las destrezas adquiridas en la actividad por estudiantes.

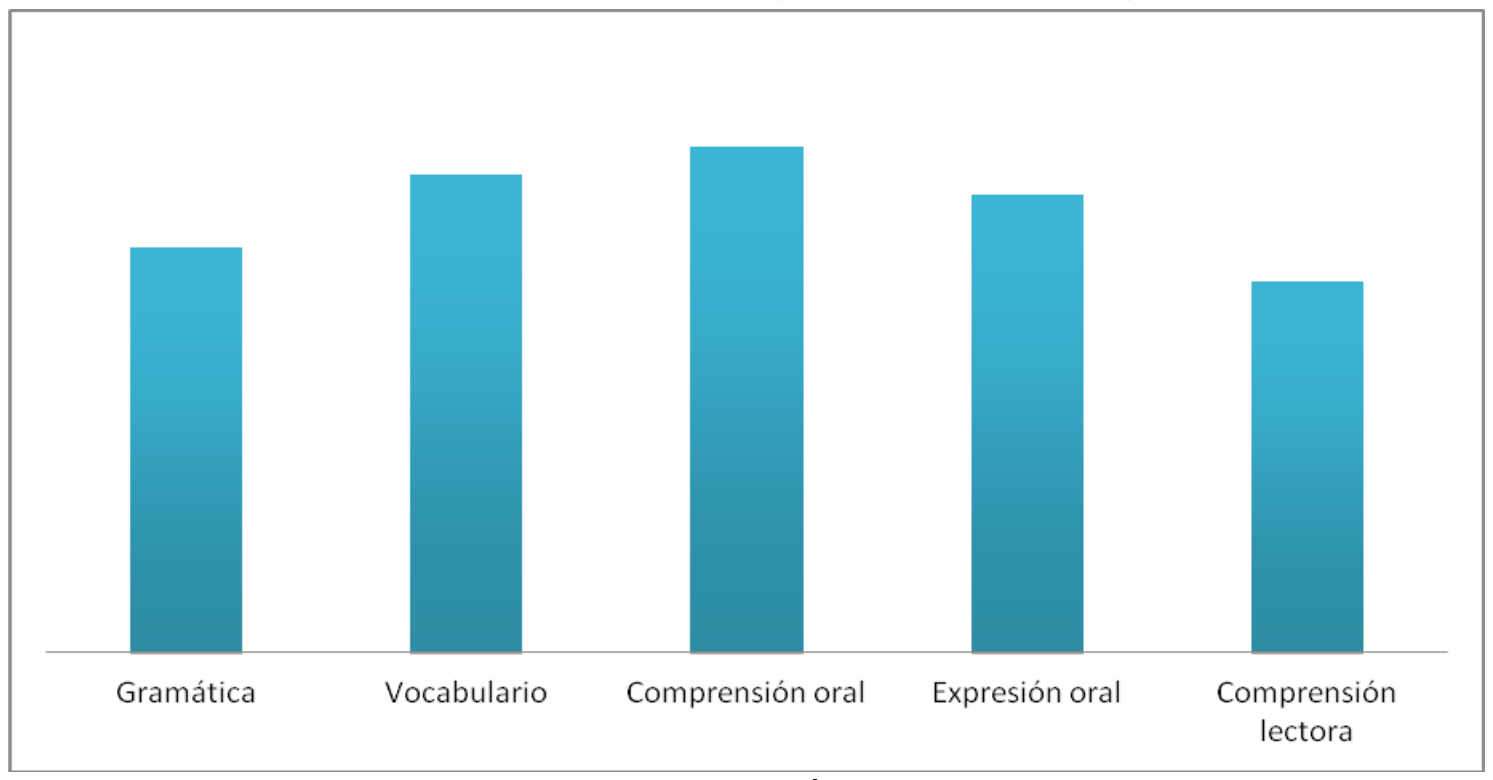

Fuente: elaboración propia. 
Tal como podemos observar en el gráfico anterior (Gráfica 3), los resultados del aprendizaje han sido muy positivos, ya que, en todas las destrezas analizadas, los sujetos afirman en porcentajes superiores al $70 \%$ que han mejorado. Atendiendo a las destrezas analizadas se ha observado que un $80 \%(\mathrm{~N}=60)$ ha perfeccionado la gramática, un 94,6 $\%(\mathrm{~N}=71)$ su vocabulario, un $90,6 \%(\mathrm{~N}=68)$ su expresión oral, un $73,3 \%(\mathrm{~N}=55)$ su comprensión lectora y un $100 \%(\mathrm{~N}=75)$ la comprensión oral.

En el caso de la gramática, un $80 \%(\mathrm{~N}=60)$ de los alumnos afirma que ha mejorado dicha competencia. Un ejemplo de ello se recoge en la afirmación:

Respecto a la gramática, me ha resultado especialmente curioso el uso de «would like» para expresar deseo que va acompañado de un infinitivo. En mi opinión, conocer este punto de gramática puede ser verdaderamente útil para la vida diaria, ya que mejora el registro del discurso y permite disponer de más recursos para expresar algo tan sencillo como puede ser el deseo de hacer algo (Estudiante A1).

En cuanto al vocabulario, el 94,6\% $(\mathrm{N}=71)$ sostiene haber aprendido muchas palabras nuevas. Un aspecto destacable consiste en que la mayoría de los participantes ha fijado su atención en palabras de uso coloquial y malsonantes, en muchas ocasiones no incluidas en la educación formal (fucking desert o fucking body). Asimismo, resulta significativo que la mayoría de ellos haya observado las palabras que han utilizado los subtituladores profesionales para traducir los sonidos no verbales. En palabras de un alumno:

Siempre hay vocabulario nuevo que aprender, pero algo que me está llamando la atención es el uso de los adjetivos y verbos atribuidos a la música y los sonidos en los subtítulos. Por poner algunos ejemplos: pump clicking, spray hissing o instrumental theme music playing (Estudiante A2).

La comprensión oral es quizás el resultado más llamativo, ya que el $100 \%(\mathrm{~N}=75)$ de los encuestados afirma haber mejorado esta competencia. Esta capacidad comunicativa no solo abarca la pronunciación, sino también conocimientos socioculturales y pragmáticos. Uno de los aspectos más positivos de los buenos resultados obtenidos por esta competencia entre los participantes es que gracias a ella podrán resolver fallos conversacionales u organizar y estructurar el discurso de forma coherente. Esto será, además, muy beneficioso para afrontar mejor las asignaturas de interpretación simultánea o consecutiva que requieren un alto dominio de la comprensión oral y de estrategias comunicativas.

En el caso de la expresión oral, 68 estudiantes afirman haberla mejorado, lo que supone el 90,6\%. Estos resultados confirman las afirmaciones de Caimi (2006) en las que se sostiene que ver programas en versión original mejora esta competencia y la enriquece, ya que el propio estudiante, puede observar la entonación, el énfasis, la velocidad con la que se enuncia el mensaje, así como otros aspectos paralingüísticos más complejos como el significado de lo que se está escuchando. Una de las posibles causas para que la mejora de la expresión oral no alcance la totalidad de la muestra es que algunos participantes pueden tener un nivel más bajo de dominio de las denominadas destrezas activas.

Por último, los resultados referentes a la comprensión lectora muestran que un $73,3 \%$ de los participantes consideran que la han mejorado $(\mathrm{N}=55)$. Estos datos son 
consistentes con las investigaciones previas (BRAVO CODINHO, 2008). A pesar de ser la competencia en la que un mayor número de participantes afirman no haber experimentado mejora alguna, los resultados siguen siendo bastante positivos. Al igual que sucede en el caso de la expresión oral, este resultado puede guardar relación con el nivel lingüístico de los sujetos.

En general, lo datos referidos a la evolución de las competencias lingüísticas son muy positivos. En términos generales, el $100 \%$ de la muestra considera que sus destrezas lingüísticas han mejorado. Si bien es cierto que existen diferencias entre cada uno de los participantes, todos ellos han mejorado al menos tres competencias en particular, ya sea en menor o mayor grado. En un análisis más detallado destaca la evolución en cuanto a la adquisición de vocabulario, en la que el $100 \%$ de la muestra afirma haberla perfeccionado. En este aspecto cabe destacar el alejamiento de lo académico y la cercanía a elementos culturales coloquiales e incluso a términos despectivos 0 malsonantes. En los usos gramaticales resalta el dominio señalado por varios participantes de aspectos complejos como las oraciones pasivas o subordinadas, a pesar de haber estudiado estos puntos gramaticales siguiendo métodos tradicionales durante su formación. Igualmente, los resultados referidos a la expresión oral y a la comprensión oral son muy alentadores.

\title{
4.3 Interés
}

La última dimensión del instrumento de medida estaba destinada a evaluar el interés y la motivación despertados a raíz de la realización de la actividad. El 95 \% (N=71) de los sujetos afirma estar satisfecho con la actividad y la considera de utilidad y muy positiva para el aprendizaje de segundas lenguas. En palabras de un alumno:

\begin{abstract}
Me parece una buena manera de aprender sin realizar un esfuerzo que pueda resultar pesado, además de ser una buena forma de optimizar el tiempo, ya que mientras dedicas tiempo al ocio, sin darte cuenta te estás familiarizando con la lengua extranjera de una manera no solo oral, sino también más puramente lingüista por la información que la aplicación te ofrece (Estudiante A3).
\end{abstract}

De hecho, un elevado número de participantes respondió que había aumentado su sensibilización con la comunidad sorda y su grado de concienciación en cuanto a la necesidad de que este colectivo pueda acceder al conocimiento igual que los normoyentes e incluso llegó a considerar este tipo de traducción multimodal accesible como una futura salida laboral.

\section{Conclusiones}

Tras décadas de investigación e innovación en el campo de la enseñanza de segundas lenguas, la coyuntura actual presenta unas características tecnológicas que son óptimas para enriquecer el proceso de aprendizaje. Estas circunstancias benefician la experimentación con nuevas metodologías y su unión con las herramientas tecnológicas que ya forman parte de la cotidianidad de gran parte de la población. Así, la unión de la pujante metodología de enseñanza de segundas lenguas CLIL con la subtitulación para sordos y el uso contenido audiovisual en streaming y de las aplicaciones que ponen a 
disposición de los usuarios ha demostrado ser una buena manera de mejorar la formación del alumnado en cuanto a sus competencias tecnológicas, lingüísticas y su motivación e interés.

Las competencias lingüísticas de los participantes mejoraron notablemente y tras la realización del ejercicio se alcanzaron unos resultados muy satisfactorios.

Asimismo, se ha comprobado que gracias a la aplicación Language Learning with Netflix y el visionado de dos episodios de una serie con subtitulado para sordos mejora el interés y la motivación del alumnado de educación superior para aprender una segunda lengua. Los sujetos han mostrado un gran interés en el aprendizaje de la lengua inglesa en contextos reales y coloquiales, y se vieron enormemente motivados al usar aplicaciones de nuevas tecnologías.

Dentro de las virtudes de este estudio se puede contar con que ha servido para fomentar el aprendizaje autónomo y para y sensibilizar al alumnado con la comunidad sorda. Con su participación, los sujetos se han visto abocados a la reflexión sobre su propio proceso de aprendizaje y sobre la importancia de seguir utilizando métodos que les permitan aprender de forma autónoma y que les resulten interesantes. Igualmente, importante resulta la difusión de una modalidad de traducción a veces desconocida para las generaciones más jóvenes, a pesar de ser usuarios frecuentes de contenido audiovisual subtitulado. Otro aspecto importante es que el uso reflexivo de la subtitulación para sordos sirve para ser más consciente de las necesidades de la comunidad sorda y todos se han sensibilizado más para con este grupo de usuarios.

Resulta importante destacar que esta investigación queda restringida a las percepciones de los participantes sobre el grado de motivación y la mejora de sus propias competencias lingüísticas. La consecución lógica de este estudio pasaría por examinar el rendimiento académico de los sujetos con pruebas de tipo pre-test y post-test con grupo de control y experimental que permita comprobar si realmente la metodología utilizada mejora las competencias, más allá de la estadística descriptiva.

A la vista de los hallazgos encontrados y a pesar del tamaño de la muestra, esta experiencia piloto ha resultado de gran utilidad y corrobora las investigaciones de varios autores que apuestan por la inclusión de la subtitulación en la enseñanza de segundas lenguas. Se debe considerar que los contenidos docentes, si se pretende aumentar el interés del alumnado, deben ser actuales e integrar la cotidianidad tecnológica de los alumnos. Esto pasa necesariamente por una actualización de las metodologías docentes. Desde aquí se aboga por su uso en entornos universitarios y académicos y por la realización de tareas específicas de acuerdo con los objetivos de aprendizaje que se indican en las guías docentes de las diferentes asignaturas de enseñanza de lenguas extranjeras. No obstante, el uso de contenido audiovisual en streaming junto con aplicaciones de subtitulado para sordos y siguiendo las premisas de la metodología CLIL abre un camino que puede ser de gran interés para el ámbito educativo.

\section{Reconocimientos}

El presente texto nace en el marco del proyecto AL-MUSACTRA "Acceso universal a museos andaluces a través de la traducción" (B-TIC-352-UGR18. Junta de Andalucía). 


\section{Referencias}

AGENCIA AFP. Netflix alcanza 167 millones de suscripciones, pero vislumbra crecimiento más lento. Diario El Comercio, 2020. Disponible en: https://www.elcomercio.com/tendencias/netflix-aumento-suscriptores-crecimientocompetencia.html. Acceso en: 7 abr. 2020.

ALBIO, G. Un análisis preliminar de las actividades tic presentes en los libros didácticos escogidos por los PNLD 2011 y 2012 para la enseñanza de E/LE a brasileños. Texto Livre: Linguagem e Tecnologia, v. 4, n. 1, p. 24-38, 2011. Disponible en: http://periodicos.letras.ufmg.br/index.php/textolivre. Acceso en: 9 abr. 2020.

BIRD, S. A.; WILLIAMS, J. N. The effect of bimodal input on implicit and explicit memory: an investigation into the benefit of within language subtitling. Applied Psycholinguistics, 23, p. 509-533, 2002.

BRAVO CODINHO, M. D. C. Putting the reader in the picture: Screen translation and foreign language learning. 2008. 257 p. Tesis doctoral. Universitat Rovira e Virgili, 2008.

CAIMI, A. Audiovisual translation and language learning: the promotion of intralingual subtitles. The Journal of Specialised Translation, 6, p. 85-98, 2006.

CONSEJO EUROPEO. Marco Europeo Común de Referencia para las Lenguas: Aprendizaje, Enseñanza, Evaluación. (Traducido al Español por el Instituto Cervantes, 2002). Disponible en: https://cvc.cervantes.es/ensenanza/biblioteca_ele/marco/cvc_mer.pdf. Acceso en: 6 jun. 2020.

COYLE, D. CLIL - a pedagogical approach. En: VAN DEUSEN-SCHOLL, N.; HORNBERGER, N. Encyclopedia of Language and Education. Berlín: Springer, 2008. p. 97-111.

DÍAZ-CINTAS, J. Los subtítulos y la subtitulación en clase de la lengua extranjera. Abehache Revista da Associação Brasileira de Hispanistas, 3, p. 95-114, 2012.

DURÁN-MARTÍNEZ, R.; BELTRÁN-LLAVADOR, F.; MARTÍNEZ-ABAD, F. A contrastive analysis between novice and expert teachers' perceptions of school bilingual programmes. Culture and Education, v. 28, n. 4, p. 738-770, 2016. DOI: http://doi.org/10.1080/11356405.2016.1237339.

FERNÁNDEZ-COSTALES, A. Subtitling in CLIL: promoting bilingual methodologies through audiovisual translation. En: CNIIE (Ed.). Bilingual Education: Educational Trends and Key Concepts. Madrid: Ministerio de Educación, Cultura y Deporte, 2017. p. 185-196.

FRESCO, V. Estados Unidos de América: Netflix, 2017. Serie de televisión.

HUNT-GÓMEZ, C. La subtitulación como recurso didáctico en el aula CLIL. Análisis de concepciones del profesorado en formación. En: AZNAR DÍAZ, I.; CÁCERES RECHE, M. P.; ROMERO RODRÍGUEZ, J. M.; MARÍN MARÍN, J. A. (eds.). Investigación e Innovación 
Educativa. Tendencias y Retos. Madrid: Dykinson, 2020. p. 269-282.

MARTÍNEZ MARTÍNEZ, S. La audiodescripción (AD) como herramienta didáctica: Adquisición de la competencia léxica. En: CRUCES COLADO, S.; DEL POZO TRIVIÑO, M.; LUNA ALONSO, A.; ÁlVAREZ LUGRÍS, A. (eds.). Traducir en la frontera. Granada: Atrio, 2012. p. 87-102.

MARTÍNEZ MARTÍNEZ, S.; ÁLVAREZ DE MORALES MERCADO, C. Nuevas herramientas de Traducción. El SpS en la enseñanza de la traducción literaria. En: ORTEGA ARJONILLA, E.; MARTÍNEZ LÓPEZ, A. B.; GARCÍA LUQUE, F. (eds.). Cartografía de la traducción, la interpretación y las industrias de la lengua. Granada: Comares, 2017. p. 427-438.

ORGANIZACIÓN MUNDIAL DE LA SALUD (OMS). Sordera y pérdida de la audición. Recuperado de: https://www.who.int/es/news-room/fact-sheets/detail/deafness-andhearing-loss. Acceso en: 5 de mayo de 2020.

TALAVÁN ZANÓN, N.; ÁVILA CABRERA, J. First insights into the combination of dubbing and subtitling as L2 didactic tools. En: GAMBIER, Y.; CAIMI, A.; MARIOTTI, C. (eds.). Subtitles and Language Learning. Berna: Peter Lang, 2015. p. 149-172.

TALAVÁN ZANÓN, N.; ÁVILA-CABRERA, J. J.; JORDANO DE LA TORRE, M.; RODRÍGUEZ ARANCÓN, P.; COSTAL CRIADO, T; LERTOLA, J.; GONZÁLEZ VERA, P.; HORNERO CORISCO, M.; SOKOLI, S. SÁNCHEZ REQUENA, A; CALDUCH, C.; ALONSO PÉREZ, R. Subtítulos para sordos como herramienta para mejorar las destrezas orales y escritas en el aprendizaje de lenguas extranjeras. En: MARTÍN CUADRADO, A. M.; JUAN OLIVA, E.; CARRIEDO LÓPEZ, N. (coords.). Actas VIII Jornadas de Redes de Investigación en Innovación Docente. UNED: Los trabajos de fin de carrera (TFG y TFM): El camino de la profesionalización. Madrid: Universidad Nacional de Educación a Distancia, 2017. p. 222-232.

TALAVÁN ZANÓN, N. Creative Audiovisual Translation Applied to Foreign Language Education: A Preliminary Approach. JAT, Journal of Audiovisual Translation, v. 2, n, 1, p. 53-74, 2019.

VANDERPLANK, R. The value of teletext subtitles in language learning. ELT Journal, v. 42, n. 4, p. 272-281, 1988. DOI: http://doi.org/10.1093/elt/42.4.272.

Recebido em dia 24 de agosto de 2020. Aprovado em dia 22 de outubro de 2020. 